\title{
Sleep Disturbances and Predictive Factors in Caregivers of Patients with Mild Cognitive Impairment and Dementia
}

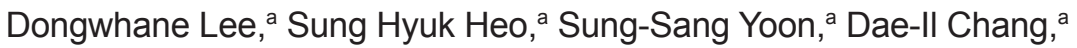 \\ Sangeui Lee, ${ }^{a}$ Hak-Young Rhee, ${ }^{b}$ Bon D. Ku, ${ }^{c}$ Key-Chung Park ${ }^{a}$ \\ aDepartment of Neurology, Kyung Hee University Medical Center, Kyung Hee University School of Medicine, Seoul, Korea \\ bDepartment of Neurology, Kyung Hee University Hospital at Gangdong, Kyung Hee University School of Medicine, Seoul, Korea \\ 'Department of Neurology, Kwandong University College of Medicine, Incheon, Korea
}

Received October 15, 2013

Revised May 12, 2014

Accepted May 13, 2014

\section{Correspondence}

Key-Chung Park, MD, PhD

Department of Neurology,

Kyung Hee University

Medical Center,

Kyung Hee University

School of Medicine,

23 Kyungheedae-ro,

Dongdaemun-gu,

Seoul 130-872, Korea

Tel $+82-2-958-8447$

Fax $+82-2-958-8490$

E-mail kcpark@khu.ac.kr
Background and Purpose We examined the characteristics of sleep disturbances and sleep patterns in the caregivers of patients with amnestic mild cognitive impairment (aMCI) and dementia.

Methods We prospectively studied 132 patients (60 with aMCI and 72 with dementia) and their caregivers, and 52 noncaregiver controls. All caregivers and controls completed several sleep questionnaires, including the Pittsburgh Sleep Quality Index (PSQI). The patients were administered neuropsychological tests and the neuropsychiatric inventory to evaluate their behavioral and neuropsychiatric symptoms of dementia (BPSD).

Results The PSQI global score was $6.25 \pm 3.88$ (mean \pm SD) for the dementia caregivers and $5.47 \pm 3.53$ for the aMCI caregivers. The Insomnia Severity Index (ISI) and short form of the Geriatric Depression Scale (GDS-S) predicted higher PSQI global scores in aMCI caregivers, and higher scores for the ISI, Epworth Sleepiness Scale (ESS), and GDS-S in dementia caregivers. BPSD, including not only agitation, depression, and appetite change in dementia patients, but also depression, apathy, and disinhibition in aMCI patients, was related to impaired sleep quality of caregivers, but nighttime behavior was not. Age and gender were not risk factors for disturbed sleep quality.

Conclusions Dementia and aMCI caregivers exhibit impaired quality of sleep versus noncaregivers. ISI, GDS-S, and ESS scores are strong indicators of poor sleep in dementia caregivers. In addition, some BPSD and parts of the neuropsychological tests may be predictive factors of sleep disturbance in dementia caregivers.

J Clin Neurol 2014;10(4):304-313

Key Words sleep disturbance, caregiver, Alzheimer's disease, vascular dementia, mild cognitive impairment.

\section{Introduction}

Behavioral and psychological symptoms of dementia (BPSD), including delusion, hallucination, agitation, depression, anxiety, apathy, irritability, aberrant motor behavior, sleep disturbances, and disinhibition, are common phenomena in patients

(a) This is an Open Access article distributed under the terms of the Creative Commons Attribution Non-Commercial License (http://creativecommons.org/licenses/by-nc/3.0) which permits unrestricted non-commercial use, distribution, and reproduction in any medium, provided the original work is properly cited. with dementia. ${ }^{1,2}$ Various types of sleep disturbance are seen frequently among these symptoms, such as obstructive sleep apnea (OSA), rapid-eye-movement sleep behavior disorder, and insomnia. ${ }^{3}$ Many caregivers of dementia patients with sleep disturbance also have sleep problems, and this is increasingly becoming a public health issue. Indeed, several cross-sectional studies have found that approximately two-thirds of older caregivers suffer from sleep disturbances ${ }^{4-6}$ and that these sleep problems are often related to the nighttime behaviors of the care recipients. Moreover, these sleep problems represent significant physical and psychological burdens to caregivers 
and are one of the major causes of patients entering nursing homes or related institutions. ${ }^{7}$ Recent studies suggest that poor caregiver sleep is linked to lowered quality of life and cognitive function, and an increased risk of the morbidity and mortality related to depression and cardiovascular disease. ${ }^{8}$

Mild cognitive impairment (MCI) is commonly applied to individuals who are not cognitively normal for their age and yet do not have overt dementia. It has been established that about $80 \%$ of patients with MCI progress to dementia within 6 years, demonstrating a higher incidence than in normal elderly. ${ }^{9}$ Despite some discrepancies in the findings of previous studies, there is growing evidence that MCI patients exhibit BPSD-and especially depression, agitation, apathy, irritability, and anxiety-associated with executive dysfunction. ${ }^{10,11}$ In addition, it has been reported that MCI caregivers are at increased risk of emotional, physical, and social burden, as are the caregivers of patients with Alzheimer's disease (AD). ${ }^{12,13}$

It is important to recognize sleep problems in caregivers and to provide appropriate interventions to improve their health outcomes. However, there have been no studies of sleep patterns and sleep disturbances in caregivers of patients with $\mathrm{MCI}$ or dementia in a Korean population. In addition, little is known about how the neuropsychiatric features found in MCI influence the sleep quality of caregivers. Accordingly, the aims of the present study were to elucidate the sleep characteristics and disturbances in the caregivers of clinic-based patients with dementia or MCI, in order to determine whether there is any relationship between BPSD in patients with dementia and the quality of sleep experienced by their caregivers.

\section{Methods}

\section{Study participants}

Caregivers of amnestic MCI (aMCI) or dementia patients ( $n=$ 132; 60 with aMCI, 72 with dementia), and 56 noncaregiver controls were recruited consecutively from the Department of Neurology at Kyung Hee University Medical Center between January 2011 and June 2013. Patients and caregivers who did not complete the tests or questionnaires were excluded. The patients had been diagnosed with aMCI, probable $\mathrm{AD}$, or probable vascular dementia (VD) by a neurologist. MCI was diagnosed according to the criteria proposed by Petersen. ${ }^{14}$ Among the subtypes of MCI, only patients with aMCI were enrolled. Both single-and multiple-domain aMCI were included. The patients with $\mathrm{AD}$ met the "probable $\mathrm{AD}$ " criteria proposed by the National Institute of Neurological and Communicative Disorders and Stroke-the Alzheimer's Disease and Related Disorders Association. ${ }^{15}$ Patients with VD fulfilled the probable or possible criteria of the National Institute of Neurological Disorders and Stroke and Association International pour la
Recherché et l'Enseignement en Neurosciences. ${ }^{16}$

The inclusion criteria for caregivers were as follows: 1) 40 years of age or older, 2) caring for a patient with a diagnosis of $\mathrm{AD}, \mathrm{VD}$, or aMCI in the caregiver's home, and 3) providing at least $10 \mathrm{~h}$ of care per week. The exclusion criteria for caregivers were any known cause of cognitive decline, such as neurodegenerative disorders, cerebrovascular diseases, psychiatric disorders, metabolic derangements, or current major illness, as follows: 1) cognitive decline due to a neurodegenerative disorder, such as Parkinson's disease with dementia, frontotemporal dementia, Huntington's disease, Creutzfeldt-Jakob disease, or hydrocephalus, 2) cognitive impairment due to metabolic derangement, including vitamin deficiency, thyroid disease, or neurosyphilis, 3) mixed dementia satisfying the diagnostic criteria of $\mathrm{AD}$ as well as having small-vessel disease on a brain magnetic resonance image, 4) current major illness (e.g., malignancy, clinically significant hepatic, cardiac, renal, or pulmonary diseases), or 5) conditions that could affect sleep disturbance (previous or current psychiatric illness, history of psychiatric drug, rhinolaryngological disease such as paranasal sinusitis, or alcohol abuse).

The noncaregiver controls were clinic-based persons not providing care to a household member. They were recruited in the same proportions of gender and age from a health-promotion center at the same hospital.

\section{Measures}

\section{Patient assessment}

Participants with dementia or MCI provided demographic characteristics including age, gender, and educational status. All patients underwent a standardized neuropsychological evaluation, the Seoul Neuropsychological Screening Battery (SNSB). ${ }^{17}$ This battery includes tests that assess the following characteristics:

1) Attention (forward and backward digit span and letter cancellation test).

2) Language and related functions (spontaneous speech, comprehension, repetition, confrontational naming using the Korean version of the Boston Naming Test, ${ }^{18}$ writing, reading, calculation, four elements of Gerstmann syndrome, and ideomotor and buccofacial praxis).

3) Visuospatial functions [interlocking pentagon drawing and the Rey-Osterrieth Complex Figure Test (RCFT), copying].

4) Verbal memory [three-word registration and recall, and the Seoul Verbal Learning Test, immediate recall (IR), 20min delayed recall (DR), and recognition].

5) Visual memory (RCFT, IR, 20-min DR, and recognition).

6) Frontal and executive function [motor impersistence, contrasting program, go-no-go test, fist-edge-palm test, alter- 
nating square and triangle, Luria loop, the phonemic and semantic-animals (A) and supermarket (S), Controlled Oral Word Association Test (COWAT), and the Stroop test (word and color reading of 112 items)].

The general cognitive status and severity of dementia were evaluated using the Korean Mini-Mental State Examination (K-MMSE), ${ }^{19}$ the Clinical Dementia Rating (CDR) scale, ${ }^{20}$ and the sum of the box score of the CDR. The scores for physical activities of daily living were estimated using the Barthel Activities of Daily Living (B-ADL) index. ${ }^{21}$ We also used the Seoul Instrumental Activities of Daily Living (S-IADL) to assess the instrumental everyday activities of the elderly. ${ }^{22}$ The Korean version of the Geriatric Depression Scale (GDS) was used to assess the level of depressed mood in patients. ${ }^{23} \mathrm{Neu}-$ ropsychiatric derangement was estimated using the Korean version of the Neuropsychiatric Inventory (K-NPI) in patients with dementia or MCI. ${ }^{24} \mathrm{~K}-\mathrm{NPI}$ is a convenient instrument that evaluates both the frequency and severity of abnormal behaviors, including delusions, hallucinations, agitation, depression, anxiety, euphoria, apathy, disinhibition, irritability, and aberrant motor behavior, as well as neurovegetative changes, including nighttime behavior and eating changes over the past 30 days. All patients underwent a comprehensive neuropsychological and functional evaluation carried out by a skilled clinical psychology practitioner who was blinded to the study hypotheses.

\section{Caregiver and control group assessment}

We collected standard demographic information on caregivers and controls (e.g., age, gender, years of education, and caregiver relationship to the patient). They were provided with a list of 15 health problems and were asked to indicate whether a physician had informed them that they currently have or had ever had each one. Subjective sleep quality was measured using the Korean version of the Pittsburgh Sleep Quality Index (PSQI), ${ }^{25}$ which assesses critical elements of the sleep experience on seven subscales: subjective sleep quality, sleeponset latency, sleep duration, sleep medication use, sleep efficiency, sleep disturbances, and daytime dysfunction (cutoff score $>6$ ). The sum of subscale scores yields the global sleep score, which ranges from 0 to 21 ; scores of $>5$ distinguish good sleepers from poor sleepers. Cronbach's alpha in the study was 0.732 . We also used the Korean version of the Epworth Sleepiness Scale (ESS) ${ }^{26}$ to evaluate daytime sleepiness, the Insomnia Severity Index (ISI) ${ }^{27}$ to evaluate the presence of insomnia (cutoff score $>7$ ), and the Berlin Questionnaire $(\mathrm{BQ})^{28}$ for OSA. The diagnosis of restless legs syndrome (RLS) was based on the diagnostic criteria of the International Restless Legs Syndrome Study Group. ${ }^{29}$ The short form of the GDS (GDS-S) was used to assess the level of depressed mood in caregivers (cutoff score $>7$ ).$^{30}$ The caregivers also completed the Korean version of the Montreal Cognitive Assessment (MOCA-K) to evaluate general cognitive function. ${ }^{31}$

All subjects provided written informed consent to participate. The protocol was approved by the Kyung Hee University Medical Center Institutional Review Board.

\section{Statistical analysis}

Statistical analyses were performed using the SPSS software (ver. 18.0 for Windows, SPSS Inc., Chicago, IL, USA). The cutoff for statistical significance was set at $p<0.05$ (two-tailed). The $t$-test was used to compare the demographic and clinical characteristics between patients with aMCI and dementia. The mean scores for caregiver ratings and participant assessment variables for the different groups (aMCI and dementia caregivers and noncaregivers) were analyzed. One-way analyses of variance (ANOVAs) were used to detect age and education differences between the groups, and $\chi^{2}$ significance tests were used to detect between-group differences in gender, RLS, and high OSA risk. Multivariate ANOVA (MANOVA) was used to examine differences in mean PSQI global scores between the groups, while Tukey's honest significant difference test was used for post-hoc comparisons. Product-moment correlation coefficients (Pearson's $r$ ) were computed to determine associations among demographic variables, quality of sleep experienced by caregivers, and other predictor variables. Multiple regression analysis with stepwise selection was implemented to determine abnormalities in cognitive function tests in each domain. The data are presented as mean $\pm \mathrm{SD}$ values, and the cutoff for statistical significance was set at $p<0.05$. This process enabled us to determine any significant predictor among the demographic and clinical characteristics.

\section{Results}

\section{Baseline characteristics of the patients}

The demographic characteristics of the patients with aMCI or dementia, including the results of $t$-tests, are given in Table 1. Patients with aMCI were younger (70.22 \pm 6.47 years vs. $74.83 \pm 6.29$ years, $t=-4.14$ ) and more educated (duration of education: $6.89 \pm 4.32$ years vs. $5.27 \pm 4.94$ years, $t=1.98$ ) than patients in the dementia group. As might be expected based on the selection criteria for the three groups, there were significant differences among the groups with respect to K-MMSE, CDR, B-ADL, and S-IADL scores, and all items of the SNSB (not presented in Table 1). The dementia group was more depressed than the aMCI group. 


\section{Baseline characteristics of the caregivers and the noncaregiver controls}

The caregivers were aged $58.14 \pm 11.62$ years. Although the caregivers were quite well-educated, the noncaregiver controls were more educated and obtained significantly higher scores on the MOCA-K. 51.7\% of the patients with aMCI were cared for by their spouses, while $38.0 \%$ of the dementia patients were. There was no difference between aMCI caregivers and dementia caregivers in terms of MOCA-K scores. The GDS-S score was significantly higher for the dementia caregivers $(4.33 \pm 3.53)$ than for the control group $(2.33 \pm 2.94)$. Caregivers did not differ from controls with respect to the number of health problems (Table 2).

\section{Comparisons of characteristics and sleep quality among aMCl and dementia caregivers and the noncaregiver controls}

A one-way ANOVA was carried out on the sleep scales, including ESS, ISI, RLS, BQ, and daytime sleep time. MANOVA was also conducted for each component score and the global PSQI score. Post-hoc analysis revealed that compared to noncaregivers, dementia caregivers obtained higher ISI scores $(\mathrm{F}=4.003, p=0.020)$, lower PSQI components of subjective sleep quality $(\mathrm{F}=6.519, p=0.002)$, longer sleep latency $(\mathrm{F}=6.880, p=0.001)$, shorter sleep duration $(\mathrm{F}=4.004, p=0.020)$, and took more sleep medication $(\mathrm{F}=3.465, p=0.033)$ (Table 3). On the other hand, aMCI caregivers exhibited lower sleep efficiency $(\mathrm{F}=4.193, p=0.017)$ than their noncaregiver counterparts. The PSQI global score was significantly higher for both the aMCI and dementia caregivers $(\mathrm{F}=8.148, p<0.001)$ than for noncaregivers, but did not differ significantly between aMCI and dementia caregivers.

\section{Association between caregivers' relationship with their patient and sleep characteristics}

Subgroup analyses between caregivers' relationships were performed, with the exception of three caregivers (two aMCI and one dementia caregivers) who were siblings of the patients (Table 4). The offspring group included sons, sons-inlaw, daughters, and daughters-in-law. The sleep latency was longer and the sleep efficiency was lower in the spouse group than in the offspring group. The PSQI global score was significantly higher in the aMCI spouse group $(6.53 \pm 3.69)$ than in the offspring group $(4.57 \pm 3.10)$, but this was not the case

Table 1. Demographics of patients with aMCl or dementia

\begin{tabular}{lcccc}
\hline & Patients with aMCl $(n=60)$ & Patients with dementia $(n=72)$ & $t$ & $p$ \\
\hline Age & $70.22 \pm 6.47$ & $74.83 \pm 6.29$ & -4.14 & $<0.001$ \\
Gender (female), \% & 71.7 & 56.9 & 3.07 & 0.080 \\
Education, years & $6.89 \pm 4.32$ & $5.27 \pm 4.94$ & 1.98 & 0.050 \\
K-MMSE & $24.82 \pm 3.81$ & $16.12 \pm 6.80$ & 9.25 & $<0.001$ \\
CDR & $0.45 \pm 0.18$ & $1.01 \pm 0.61$ & -7.41 & $<0.001$ \\
CDR-SB & $1.61 \pm 1.27$ & $6.11 \pm 3.81$ & -9.43 & $<0.001$ \\
B-ADL & $19.87 \pm 0.91$ & $18.57 \pm 3.22$ & 3.27 & 0.002 \\
S-IADL (present) & $4.57 \pm 4.42$ & $19.39 \pm 10.85$ & -10.58 & $<0.001$ \\
S-IADL (potential) & $4.28 \pm 4.13$ & $18.71 \pm 10.61$ & -10.61 & $<0.001$ \\
GDS & $16.1 \pm 6.71$ & $18.90 \pm 6.31$ & -2.47 & 0.015 \\
\hline Data a given & & &
\end{tabular}

Data are given as means \pm SD or percentages.

aMCl: amnestic mild cognitive impairment, B-ADL: Barthel Activities of Daily Living index, CDR: Clinical Dementia Rating, CDR-SB: the sum of the box score of the CDR, GDS: Geriatric Depression Scale, K-MMSE: Korean Mini-Mental State Examination, S-IADL: Seoul-Instrumental Activities of Daily Living.

Table 2. Characteristics of caregivers of patients with dementia or $\mathrm{aMCl}$ and non-caregiver controls

\begin{tabular}{lcccc}
\hline & $\mathrm{aMCl}$ caregiver $(n=60)$ & Dementia caregiver $(n=72)$ & Non-caregiver controls $(n=56)$ & $\mathrm{F}$ \\
\hline Age, years & $58.50 \pm 14.26$ & $58.05 \pm 11.75$ & $57.87 \pm 7.89$ & 6.045 \\
Gender (female), \% & 51.7 & 65.3 & 60.7 & $13.56 \pm 4.57^{\mathrm{a}}$ \\
Education, years & $11.40 \pm 4.48^{\mathrm{b}}$ & $10.22 \pm 4.27^{\mathrm{b}}$ & $0.59 \pm 0.80$ & $8.968^{*}$ \\
Number of health problems & $0.73 \pm 0.84$ & $0.91 \pm 0.93$ & 2.296 \\
MOCA-K & $25.28 \pm 4.54^{\mathrm{b}}$ & $25.51 \pm 3.13^{\mathrm{b}}$ & $2.03 \pm 2.84^{\mathrm{a}}$ & $4.146^{\ddagger}$ \\
GDS-S & $3.68 \pm 3.50^{\mathrm{ab}}$ & $4.33 \pm 3.53^{\mathrm{a}}$ & $2.33 \pm 2.94^{\mathrm{b}}$ & $5.639^{\dagger}$ \\
\hline
\end{tabular}

Data are given as means \pm SD or percentages. $a>b$, Tukey's honestly significance difference post hoc testing was performed to assess for differences among subgroups.

${ }^{*} p<0.001,{ }^{\dagger} p<0.01,{ }^{\ddagger} p<0.05$.

aMCl: amnestic mild cognitive impairment, GDS-S: short form of Geriatric Depression Scale, MOCA-K: Montreal Cognitive AssessmentKorean version. 
for the dementia spouse group.

\section{Relationships between PSQI and patient variables and caregiver measures}

Among the aMCI caregivers, bivariate correlations revealed a significant relationship between PSQI global score and the age of the caregiver $(r=0.283, p=0.029)$, education years of the caregiver $(r=-0.381, p=0.003)$, ISI score $(r=0.786, p<0.001)$, and K-MMSE score of the patient $(r=0.273, p=0.035)$ (Table $5)$. In the items of the SNSB, RCFT-IR $(r=0.332, p=0.010)$ and RCFT-DR $(r=0.328, p=0.011)$ scores of the aMCI patients and the COWAT-A scores $(r=0.235, p=0.047)$ of the dementia patients were significantly related to PSQI global scores (data not presented). Among dementia caregivers, ISI and GDS-S scores were correlated with both global and subjective sleep quality as measured using the PSQI.

\section{Impact of BPSD in patients on caregiver sleep quality}

The mean composite scores of the K-NPI of subscales and prevalence in the entire patient cohort are given in Table 6. Among the aMCI caregivers, depression $(r=0.279, p=0.013)$, apathy ( $r=0.261, p=0.013)$, and disinhibition $(r=0.319, p=0.013)$ were associated with high PSQI global scores (Table 5). Among the dementia caregivers, there were significant interactions between PSQI global score and agitation/aggression $(r=0.287$, $p=0.006)$, depression $(r=0.339, p=0.001)$, and appetite changes $(r=0.322, p=0.007)$. Depression $(r=0.292, p=0.013)$ and appetite changes $(r=0.324, p=0.006)$ were also associated with decreased subjective sleep quality.

\section{Variables associated with sleep disturbances}

Stepwise simultaneous multiple regression analyses were conducted to assess variables related to sleep disturbance. The dependent variable was the PSQI global score, and the independent variables were age, gender, education years, the MOCA$\mathrm{K}$, GDS-S, ESS, and ISI scores of caregivers, the K-MMSE, $\mathrm{S}$-IADL, and B-ADL scores of patients, and scores on the subscales of the K-NPI, including agitation/aggression, depression, apathy, disinhibition, and appetite changes, which are strongly correlated with BPSD. The final model explained $64.1 \%[\mathrm{~F}(2,57)=53.653, p<0.001]$ and $65.5 \%[\mathrm{~F}(3,68)=45.953$, $p<0.002]$ of the variance in PSQI global scores in the aMCI and dementia caregiver groups, respectively. The data given in Table 7 indicate that ISI $(\beta=0.696, t=8.055, p<0.001)$ and GDS-S $(\beta=0.209, t=2.417, p=0.019)$ scores predicted higher PSQI global scores in aMCI caregivers. Moreover, ISI $(\beta=0.636$, $t=8.724, p<0.001)$, ESS $(\beta=0.234, t=3.223, p=0.002)$, and GDS-S $(\beta=0.245, t=3.439, p=0.001)$ scores made independent contributions to the variance of the quality of sleep experienced by dementia caregivers.

Table 3. Comparison of means (SDs) on sleep questionnaires and the PSQI for caregivers

\begin{tabular}{|c|c|c|c|c|}
\hline & aMCl caregiver $(n=60)$ & Dementia caregiver $(n=72)$ & Controls $(n=56)$ & $\mathrm{F}$ \\
\hline ESS§ & $2.52 \pm 3.57^{b}$ & $1.79 \pm 2.82^{b}$ & $4.34 \pm 3.41^{a}$ & $9.954^{*}$ \\
\hline $10-24, n(\%)$ & $5(8.3)$ & 2 & 1 & \\
\hline RLS, n (\%)" & $1(0.0)$ & $2(1.4)$ & $2(3.6)$ & 0.203 \\
\hline$|S| \S$ & $5.22 \pm 6.36^{\mathrm{ab}}$ & $6.39 \pm 6.29 a$ & $3.43 \pm 4.67^{b}$ & 4.003 \\
\hline $\mathrm{BQ}^{\|}$high risk, n (\%) & $6(10)$ & $13(26.4)$ & $6(10.7)$ & 1.148 \\
\hline \multicolumn{5}{|l|}{ PSQI subscales" } \\
\hline 1. Subjective sleep quality & $1.13 \pm 0.72^{\mathrm{ab}}$ & $1.31 \pm 0.76^{a}$ & $0.84 \pm 0.68^{b}$ & $6.519^{\dagger}$ \\
\hline 2. Sleep latency & $1.12 \pm 1.18^{\mathrm{ab}}$ & $1.46 \pm 1.24^{a}$ & $0.70 \pm 0.99^{b}$ & $6.880^{*}$ \\
\hline 3. Sleep duration (hour) & $1.37 \pm 1.10^{\mathrm{ab}}$ & $1.53 \pm 1.06^{a}$ & $1.02 \pm 0.86^{\mathrm{b}}$ & $4.004^{\ddagger}$ \\
\hline 4. Sleep efficiency & $0.47 \pm 0.79^{a}$ & $0.36 \pm 0.76^{\mathrm{ab}}$ & $0.11 \pm 0.45^{b}$ & $4.193^{\ddagger}$ \\
\hline 5. Sleep disturbance (\%) & $0.65 \pm 0.52$ & $0.69 \pm 0.46$ & $0.71 \pm 0.43$ & 0.255 \\
\hline 6. Sleep medication & $0.18 \pm 0.65^{\mathrm{ab}}$ & $0.33 \pm 0.82^{a}$ & $0.03 \pm 0.19^{b}$ & $3.465^{\ddagger}$ \\
\hline 7. Daytime dysfunction & $0.55 \pm 0.87$ & $0.56 \pm 0.79$ & $0.38 \pm 0.59$ & 1.071 \\
\hline 8. PSQI global score & $5.47 \pm 3.53^{a}$ & $6.25 \pm 3.88^{a}$ & $3.79 \pm 2.71^{\mathrm{b}}$ & $8.148^{*}$ \\
\hline Sleep latency (min) & $28.21 \pm 25.18^{a b}$ & $35.76 \pm 34.22^{a}$ & $21.21 \pm 26.59^{b}$ & $3.907^{\ddagger}$ \\
\hline Sleep duration (hour) & $6.36 \pm 1.47$ & $6.20 \pm 1.31$ & $6.51 \pm 1.30$ & 0.955 \\
\hline Sleep efficiency (\%) & $88.41 \pm 9.75^{\mathrm{b}}$ & $89.17 \pm 9.33^{b}$ & $90.49 \pm 9.38^{a}$ & $7.625^{*}$ \\
\hline Daytime sleep time $(\mathrm{min})^{\S}$ & $20.13 \pm 34.45$ & $16.27 \pm 31.36$ & $22.98 \pm 32.85$ & 0.672 \\
\hline
\end{tabular}

Data are given as means \pm SD or percentages. $a>b$, Tukey's honestly significance difference post hoc testing was performed to assess for differences among subgroups.

${ }^{*} p<0.001,{ }^{\dagger} p<0.01,{ }^{\ddagger} p<0.05$ (two-tailed), ${ }^{\circ}$ One-way ANOVA, "Chi-squared test, "MANOVA.

aMCl: amnestic mild cognitive impairment, ANOVA: analysis of variance, BQ: Berlin Questionnaire, ESS: Epworth Sleepiness Scale, ISI: Insomnia Severity Index, MANOVA: multivariate ANOVA, min: minute, PSQI: Pittsburgh Sleep Quality Index, RLS: restless leg syndrome. 
Table 4. Comparison of means (SDs) on sleep questionnaires and the PSQI for caregivers according to relationship

\begin{tabular}{|c|c|c|c|c|}
\hline & Spouse group & Offspring group & $t$ & $p$ \\
\hline \multicolumn{5}{|c|}{ aMCl caregivers [ $n=58$, spouse group $(n=30)$, offspring group $(n=28)]$} \\
\hline Mean age & $69.77 \pm 10.52$ & $47.07 \pm 6.27$ & 9.89 & $<0.001$ \\
\hline ESS & $1.53 \pm 2.46$ & $3.75 \pm 4.27$ & -2.40 & 0.021 \\
\hline$|S|$ & $6.23 \pm 6.73$ & $4.32 \pm 6.09$ & 1.13 & 0.263 \\
\hline \multicolumn{5}{|l|}{ PSQI subscales } \\
\hline 1. Subjective sleep quality & $1.30 \pm 0.70$ & $1.00 \pm 0.72$ & 1.61 & 0.114 \\
\hline 2. Sleep latency & $1.50 \pm 1.22$ & $0.75 \pm 1.04$ & 2.50 & 0.015 \\
\hline 3. Sleep duration & $1.53 \pm 1.17$ & $1.21 \pm 1.03$ & 1.10 & 0.276 \\
\hline 4. Sleep efficiency & $0.77 \pm 0.94$ & $0.18 \pm 0.48$ & 3.05 & 0.004 \\
\hline 5. Sleep disturbance & $0.73 \pm 0.45$ & $0.61 \pm 0.57$ & 0.94 & 0.354 \\
\hline 6. Sleep medication & $0.20 \pm 0.66$ & $0.18 \pm 0.67$ & 0.12 & 0.903 \\
\hline 7. Daytime dysfunction & $0.50 \pm 0.90$ & $0.64 \pm 0.87$ & -0.61 & 0.542 \\
\hline Global score & $6.53 \pm 3.69$ & $4.57 \pm 3.10$ & 2.18 & 0.033 \\
\hline Sleep latency (min) & $33.67 \pm 27.81$ & $23.04 \pm 21.86$ & 1.61 & 0.113 \\
\hline Sleep duration (hour) & $6.17 \pm 1.52$ & $6.53 \pm 1.46$ & -0.91 & 0.367 \\
\hline Sleep efficiency (\%) & $84.27 \pm 10.39$ & $92.36 \pm 7.25$ & -3.46 & 0.001 \\
\hline Daytime sleep time (min) & $29.33 \pm 38.57$ & $11.82 \pm 28.28$ & 1.98 & 0.053 \\
\hline \multicolumn{5}{|c|}{ Dementia caregivers [ $n=71$, spouse group $(n=27)$, offspring group $(n=44)]$} \\
\hline Mean age & $68.48 \pm 9.47$ & $51.89 \pm 8.02$ & 7.90 & $<0.001$ \\
\hline ESS & $1.78 \pm 2.90$ & $1.68 \pm 2.72$ & 0.14 & 0.889 \\
\hline$|S|$ & $8.07 \pm 6.56$ & $5.14 \pm 5.80$ & 1.97 & 0.053 \\
\hline \multicolumn{5}{|l|}{ PSQI subscales } \\
\hline 1. Subjective sleep quality & $1.41 \pm 0.89$ & $1.23 \pm 0.68$ & 0.91 & 0.371 \\
\hline 2. Sleep latency & $1.81 \pm 1.33$ & $1.20 \pm 1.13$ & 2.06 & 0.043 \\
\hline 3. Sleep duration & $1.41 \pm 1.08$ & $1.59 \pm 1.06$ & -0.70 & 0.486 \\
\hline 4. Sleep efficiency & $0.67 \pm 0.96$ & $0.18 \pm 0.54$ & 2.40 & 0.022 \\
\hline 5. Sleep disturbance & $0.74 \pm 0.45$ & $0.66 \pm 0.48$ & 0.72 & 0.477 \\
\hline 6. Sleep medication & $0.52 \pm 1.01$ & $0.28 \pm 0.68$ & 1.32 & 0.194 \\
\hline 7. Daytime dysfunction & $0.63 \pm 0.88$ & $0.50 \pm 0.73$ & 0.67 & 0.505 \\
\hline Global score & $7.19 \pm 4.42$ & $5.59 \pm 3.43$ & 1.70 & 0.094 \\
\hline Sleep latency (min) & $45.46 \pm 44.16$ & $29.26 \pm 25.26$ & 1.74 & 0.090 \\
\hline Sleep duration (hour) & $6.42 \pm 1.43$ & $6.08 \pm 1.25$ & 1.05 & 0.299 \\
\hline Sleep efficiency (\%) & $85.70 \pm 11.95$ & $91.40 \pm 6.65$ & -2.27 & 0.029 \\
\hline Daytime sleep time (min) & $22.78 \pm 38.24$ & $12.56 \pm 26.19$ & 1.33 & 0.189 \\
\hline
\end{tabular}

Data are given as means \pm SD or percentages. Offspring group included sons, sons-in-law, daughters, and daughters-in-law.

aMCl: amnestic mild cognitive impairment, ESS: Epworth Sleepiness Scale, ISI: Insomnia Severity Index, min: minute, PSQI: Pittsburgh Sleep Quality Index.

\section{Discussion}

In this study we examined sleep patterns and disturbances thereof experience by the caregivers of patients with aMCI or dementia. We also investigated sleep scales, neuropsychological tests, and a neuropsychiatric inventory to assess correlations between quality of sleep and various clinical characteristics. Clinic-based patients with aMCI or dementia who had mild-to-moderate dementia were enrolled, and they would be expected to exhibit lower CDR scores and a lower prevalence of BPSD compared with institutionalized patients.
As expected, and consistent with previous studies, the caregivers of dementia patients experienced a lower quality of sleep than did noncaregivers. Interestingly, the BPSD and cognitive impairments of aMCI patients with preserved activities of daily living impinged on the sleep quality experienced by their caregivers. In addition, the PSQI global score $(6.25 \pm 3.88)$ tended to be lower than those found in previous studies of dementia caregivers, ${ }^{32,33}$ and similar to those reported for the caregivers of patients with Parkinson's disease ${ }^{34}$ However, in contrast to previous studies finding that the severity of dementia directly impacted upon the sleep disturbances experi- 
Table 5. Correlations between the Pittsburgh Sleep Quality Index (PSQI) and patient variables and caregiver measures according to caregiving group

\begin{tabular}{|c|c|c|c|c|}
\hline \multirow{2}{*}{ PSQI } & \multicolumn{2}{|c|}{$\mathrm{aMCl}$ caregivers $(n=60)$} & \multicolumn{2}{|c|}{ Dementia caregivers $(n=72)$} \\
\hline & Global sleep quality & Subjective sleep quality & Global sleep quality & Subjective sleep quality \\
\hline Caregiver age & $0.283^{\dagger}$ & $0.262^{\dagger}$ & 0.125 & 0.075 \\
\hline Caregiver education & $-0.381^{*}$ & $-0.309^{\dagger}$ & -0.087 & -0.077 \\
\hline Caregiver gender & 0.072 & 0.133 & -0.081 & -0.091 \\
\hline ESS & 0.048 & 0.006 & $0.436^{*}$ & 0.226 \\
\hline$|S|$ & $0.786^{*}$ & $0.740^{*}$ & $0.740^{*}$ & $0.710^{*}$ \\
\hline$B Q$ & 0.114 & 0.093 & 0.194 & 0.192 \\
\hline MOCA-K (caregiver) & -0.214 & -0.135 & $-0.250^{\dagger}$ & -0.202 \\
\hline GDS-S (caregiver) & $0.508^{\dagger}$ & $0.405^{*}$ & $0.396^{*}$ & $0.286^{\dagger}$ \\
\hline Patient age & -0.115 & -0.057 & -0.024 & -0.057 \\
\hline Patient education & -0.031 & 0.065 & 0.007 & 0.026 \\
\hline K-MMSE (patient) & $0.273^{*}$ & $0.328^{\dagger}$ & 0.003 & 0.066 \\
\hline CDR & 0.106 & 0.053 & 0.157 & 0.132 \\
\hline CDR-SB & 0.184 & 0.252 & 0.111 & 0.095 \\
\hline B-ADL & 0.104 & 0.027 & -0.066 & -0.078 \\
\hline S-IADL (present) & 0.143 & $0.262^{\dagger}$ & 0.184 & 0.154 \\
\hline S-IADL (potent) & 0.075 & $0.316^{\dagger}$ & 0.163 & 0.137 \\
\hline GDS (patient) & -0.007 & -0.066 & $0.395^{*}$ & 0.002 \\
\hline \multicolumn{5}{|l|}{ K-NPI } \\
\hline 1) Delusion & -0.029 & 0.043 & 0.208 & 0.222 \\
\hline 2) Hallucination & -0.092 & -0.205 & 0.008 & -0.012 \\
\hline 3) Agitation/aggression & 0.254 & 0.169 & $0.287^{\dagger}$ & 0.166 \\
\hline 4) Depression/dysphoria & $0.276^{\dagger}$ & 0.210 & $0.339 *$ & $0.292^{\dagger}$ \\
\hline 5) Anxiety & -0.001 & 0.012 & 0.182 & 0.029 \\
\hline 6) Elation/euphoria & $n / a$ & $\mathrm{n} / \mathrm{a}$ & -0.091 & -0.002 \\
\hline 7) Apathy & $0.261^{\dagger}$ & 0.244 & 0.127 & 0.073 \\
\hline 8) Disinhibition & $0.319^{\dagger}$ & 0.241 & 0.096 & 0.009 \\
\hline 9) Irritability & 0.014 & -0.054 & 0.053 & 0.012 \\
\hline 10) Aberrant motor behavior & 0.094 & 0.157 & 0.025 & -0.011 \\
\hline 11) Sleep/nighttime behavior & 0.171 & 0.104 & -0.103 & 0.038 \\
\hline 12) Appetite/eating disorder & -0.041 & -0.098 & $0.322^{*}$ & $0.324^{*}$ \\
\hline K-NPI total score & 0.236 & 0.152 & 0.204 & 0.161 \\
\hline
\end{tabular}

Values are correlation coefficients.

${ }^{*} p<0.01,{ }^{\dagger} p<0.05$ (two-tailed).

aMCl: amnestic mild cognitive disorder, B-ADL: Barthel Activities of Daily Living index, BQ: Berlin Questionnaire, CDR: Clinical Dementia Rating, CDR-SB: the sum of the box score of the CDR, ESS: Epworth Sleepiness Scale, GDS: Geriatric Depression Scale, GDS-S: short form of the Geriatric Depression Scale, ISI: Insomnia Severity Index, K-MMSE: Korean Mini-Mental State Examination, K-NPI: Korean version of Neuropsychiatric Inventory, MOCA-K: Montreal Cognitive Assessment-Korean version, n/a: not applicable, S-IADL: Seoul-Instrumental Activities of Daily Living.

enced by caregivers, we found that the PSQI global score did not vary with the CDR score. ${ }^{35,36}$ Not only were the global PSQI scores worse in dementia caregivers than in noncaregivers, but so also was the subjective sleep quality of dementia caregivers. Although polysomnography (PSG) and self-reported sleep questionnaires revealed that dementia caregivers perceive their sleep to be generally worse than that of noncaregiver controls in some studies, this perception did not correspond with objective sleep assessments. ${ }^{32,37}$ In agreement with other studies ${ }^{38}$ the present results revealed decreased sleep dura- tion in dementia caregivers, which was lower than the needs of a normal adult (7-9 h/day), and increased sleep latency. Moreover, ISI was the most powerful contributor to sleep disturbances in dementia caregivers, and $38 \%$ of patients with ISI scores of $>7$ complained of moderate-to-severe insomnia. Caregivers who are woken frequently can fall into iatrogenic sleep routines that are part of an ineffective attempt to compensate for having their nightly rest disturbed by cat-napping throughout the day, drinking coffee to stay awake, or consuming alcohol to help fall asleep, progressing eventually to a vi- 
Table 6. Mean composite scores (frequency $\times$ severity) of Korean-Neuropsychiatric Inventory (K-NPI) of subscales and prevalence in the total patients

\begin{tabular}{|c|c|c|c|c|}
\hline & \multicolumn{2}{|c|}{ Prevalence (\%) } & \multicolumn{2}{|c|}{ K-NPI score of subscale } \\
\hline & $\begin{array}{l}\text { Patients with aMCl } \\
\qquad(n=60)\end{array}$ & $\begin{array}{l}\text { Patients with dementia } \\
\qquad(n=72)\end{array}$ & $\begin{array}{l}\text { Patients with aMCl } \\
\qquad(n=60)\end{array}$ & $\begin{array}{l}\text { Patients with dementia } \\
\qquad(n=72)\end{array}$ \\
\hline 1) Delusion & 8.3 & 18.1 & $0.20 \pm 0.75$ & $0.82 \pm 2.08$ \\
\hline 2) Hallucination & 1.7 & 18.1 & $0.02 \pm 0.13$ & $0.94 \pm 2.79$ \\
\hline 3) Agitation/aggression & 13.3 & 27.8 & $0.27 \pm 1.09$ & $1.18 \pm 2.68$ \\
\hline 4) Depression/dysphoria & 43.3 & 51.4 & $0.87 \pm 1.46$ & $1.83 \pm 2.82$ \\
\hline 5) Anxiety & 20.0 & 37.5 & $0.42 \pm 1.08$ & $1.42 \pm 2.42$ \\
\hline 6) Elation/euphoria & 0.0 & 4.2 & $\mathrm{n} / \mathrm{a}$ & $0.24 \pm 1.49$ \\
\hline 7) Apathy & 30.0 & 45.8 & $0.73 \pm 1.64$ & $2.33 \pm 3.46$ \\
\hline 8) Disinhibition & 6.7 & 19.4 & $0.15 \pm 0.66$ & $0.82 \pm 1.90$ \\
\hline 9) Irritability & 28.3 & 45.8 & $0.62 \pm 1.26$ & $1.96 \pm 2.99$ \\
\hline 10) Aberrant motor behavior & 1.7 & 26.4 & $0.03 \pm 0.26$ & $1.78 \pm 3.43$ \\
\hline 11) Sleep/nighttime behavior & 20.0 & 34.7 & $0.98 \pm 2.28$ & $1.72 \pm 2.93$ \\
\hline 12) Appetite/eating disorder & 28.3 & 54.2 & $1.27 \pm 2.41$ & $3.17 \pm 3.67$ \\
\hline
\end{tabular}

Data are given as means $\pm S D$ or percentages.

aMCl: amnestic mild cognitive disorder, $\mathrm{n} / \mathrm{a}$ : not applicable.

cious cycle. $^{4}$

A novel finding of our study was that agitation/aggression, depression, and appetite changes as reflected by the K-NPI scores were significantly related to global sleep quality, whereas nighttime behavior was not. Previous studies found that the nighttime behaviors of care recipients precipitated caregiver awakening, and that it can be difficult for vulnerable caregivers to fall asleep again. ${ }^{4}$ Although nocturnal behavioral disturbances are no longer present, disrupted sleep patterns persist. ${ }^{39}$ Another finding of our study concerned the predictive effects of cognitive function on sleep quality for dementia patients and caregivers. MOCA-K was negatively associated with the quality of sleep among dementia caregivers. Furthermore, in neuropsychological tests, how a dementia patient performs in the COWAT-A task, which is included in the frontal lobe function test, is related to disturbed sleep quality in their caregiver. This may indicate that some of the aforementioned BPSD or some aspects of frontal dysfunction in dementia patients are early indicators of caregiver sleep disturbances.

Mean PSQI global scores for aMCI caregivers were not above the cutoff; however, significant differences in sleep efficiency and PSQI global score were found between these caregivers and their noncaregiver counterparts. The ISI score, which was positively associated with PSQI global score, appeared to be higher for aMCI caregivers than for noncaregivers, but the difference did not reach statistical significance. Insomnia was experienced by $29 \%$ (18/60) of the aMCI caregivers. In fact, primary sleep disorders such as OSA, RLS, and periodic limb movement disorder are more common in older adults, but they frequently go undiagnosed, and so may play unrecognized roles in many of the sleep complaints reported by caregivers. ${ }^{40}$
There is growing evidence that sleep is significantly impaired in patients with MCI at both the objective and subjective levels, and might be useful as a surrogate marker of preclinical disease. ${ }^{41}$ In terms of the impact of BPSD for patients with aMCI on sleep disturbance, it is worth noting that disinhibition, apathy, and depression in patients are correlated with the quality of sleep experienced by their caregivers. A recent study found that increased neuropsychiatric symptoms and worse executive functioning of MCI patients were related to a greater caregiver burden, and that caregivers with a greater burden reported lower life satisfaction and social support, and a greater need for support services. ${ }^{12}$ In addition, sleep disturbances in MCI patients could affect the quality of sleep experienced by their caregivers. Further studies concerning MCI caregivers are needed.

Our results differ from those of previous studies in several respects. Caregivers' depressive symptoms were not only significant contributors to the variance in caregiver sleep quality, but were also significantly correlated with the measured global quality of sleep for both aMCI and dementia caregivers. Caregivers scored below the cutoff for depressive symptoms in GDS-S in other studies. ${ }^{38}$ Nevertheless, $13 \%$ of aMCI caregivers and $17 \%$ of dementia caregivers scored above the cutoff (of 8) in the GDS-S in the present study. In one longitudinal study concerning clinic-based patients, caregiver depression and subjective burden were found to be the most powerful predictors of the onset of new caregiver sleep disturbances. ${ }^{36}$

Another point of difference of the present study is that aging and gender were not risk factors for disturbed sleep quality, in contrast to some previous studies in which the participants were limited to older females. ${ }^{38}$ Of course, most dementia 
Table 7. Standardized regression coefficients for sleep quality $(n=132)$

\begin{tabular}{|c|c|c|c|}
\hline & Model 1 & Model 2 & Model 3 \\
\hline \multicolumn{4}{|l|}{ aMCl caregivers $(n=60)$} \\
\hline Caregiver age & 0.156 & 0.132 & \\
\hline Caregiver gender & 0.025 & 0.023 & \\
\hline GDS-S (caregiver) & 0.209 & $0.209 *$ & \\
\hline ESS & 0.022 & 0.086 & \\
\hline$|S|$ & $0.786 *$ & $0.696^{\ddagger}$ & \\
\hline Agitation/aggression & 0.084 & 0.020 & \\
\hline Depression & 0.057 & 0.004 & \\
\hline Apathy & 0.022 & -0.012 & \\
\hline Disinhibition & 0.105 & 0.127 & \\
\hline Appetite changes & 0.047 & 0.040 & \\
\hline $\mathrm{R}^{2}$ & 0.618 & 0.653 & \\
\hline Adjusted $\mathrm{R}^{2}$ & 0.611 & 0.641 & \\
\hline$\Delta F$ & 93.643 & 5.843 & \\
\hline \multicolumn{4}{|c|}{ Dementia caregivers $(n=72)$} \\
\hline Caregiver age & -0.076 & -0.069 & -0.085 \\
\hline Caregiver gender & -0.151 & -0.130 & -0.085 \\
\hline GDS-S (caregiver) & 0.106 & $0.272^{\dagger}$ & $0.245^{\dagger}$ \\
\hline ESS & 0.263 & 0.234 & $0.234^{\dagger}$ \\
\hline$|S|$ & $0.740^{*}$ & $0.692^{*}$ & $0.636^{*}$ \\
\hline Agitation/aggression & 0.081 & 0.061 & -0.005 \\
\hline Depression & 0.155 & 0.109 & 0.102 \\
\hline Apathy & 0.011 & -0.022 & -0.001 \\
\hline Disinhibition & 0.016 & -0.022 & -0.047 \\
\hline Appetite changes & 0.110 & 0.106 & 0.105 \\
\hline $\mathrm{R}^{2}$ & 0.548 & 0.619 & 0.670 \\
\hline Adjusted $\mathrm{R}^{2}$ & 0.541 & 0.608 & 0.655 \\
\hline$\Delta F$ & 84.751 & 12.969 & \\
\hline
\end{tabular}

${ }^{*} p<0.001,{ }^{\dagger} p<0.01,{ }^{\ddagger} p<0.05$ (two-tailed).

aMCl: amnestic mild cognitive disorder, ESS: Epworth Sleepiness Scale, GDS-S: short form of the Geriatric Depression Scale, ISI: Insomnia Severity Index.

caregivers are older females; however, as the prevalence rates of dementia and/or MCI increase, we assume that caregivers of various ages and relationships to patients will be needed to perform caregiving duties.

In addition, the spouse caregivers in the present study complained more about sleep disturbances than did the offspring caregivers. In one cross-sectional study, family caregivers were more impaired than nonfamily caregivers with regard to sleep quality. ${ }^{42}$ We postulate that this can be attributed to differences in the role of caregiving among family members.

This study was subject to several limitations. First, it involved only a single center in Korea, which limits the generalizability of the results; multicenter studies are thus needed. Second, only clinic-based patients were enrolled, and most of patients had mild-to-moderate dementia. Furthermore, we did not investigate the pattern of nocturia and mobility among the patients, both of which could affect their caregivers' sleep. Third, we did not include nonamnestic MCI patients, who probably have other dementias, such as frontotemporal dementia, dementia with Lewy bodies, or VD. In addition, due to the small sample of caregivers for patients with VD, we could not evaluate its various subtypes or study the relationship between K-NPI score and sleep quality stratified according to dementia subtype (i.e., AD vs. VD). Fourth, we did not perform PSG or actigraphy, which would facilitate objective evaluations of caregiver sleep.

The findings of this study demonstrate that the quality of sleep is worse for caregivers of dementia and aMCI patients than for noncaregivers. ISI, depressive mood, and ESS were strong indicators of poor sleep among dementia caregivers, and some BPSD and aspects of neuropsychological test results appear to be early indicators of caregiver sleep disturbances.

\section{Conflicts of Interest}

The authors have no financial conflicts of interest.

\section{REFERENCES}

1. Finkel SI, Costa e Silva J, Cohen G, Miller S, Sartorius N. Behavioral and psychological signs and symptoms of dementia: a consensus statement on current knowledge and implications for research and treatment. Int Psychogeriatr 1996;8 Suppl 3:497-500.

2. Cummings JL, Vinters HV, Cole GM, Khachaturian ZS. Alzheimer's disease: etiologies, pathophysiology, cognitive reserve, and treatment opportunities. Neurology 1998;51(1 Suppl 1):S2-S17; discussion S65S67.

3. Winblad B, Palmer K, Kivipelto M, Jelic V, Fratiglioni L, Wahlund LO, et al. Mild cognitive impairment--beyond controversies, towards a consensus: report of the International Working Group on Mild Cognitive Impairment. J Intern Med 2004;256:240-246.

4. McCurry SM, Logsdon RG, Teri L, Vitiello MV. Sleep disturbances in caregivers of persons with dementia: contributing factors and treatment implications. Sleep Med Rev 2007;11:143-153.

5. Pruchno RA, Potashnik SL. Caregiving spouses. Physical and mental health in perspective. J Am Geriatr Soc 1989;37:697-705.

6. Wilcox S, King AC. Sleep complaints in older women who are family caregivers. J Gerontol B Psychol Sci Soc Sci 1999;54:P189-P198.

7. Hope T, Keene J, Gedling K, Fairburn CG, Jacoby R. Predictors of institutionalization for people with dementia living at home with a carer. Int J Geriatr Psychiatry 1998;13:682-690.

8. Schulz R, Martire LM. Family caregiving of persons with dementia: prevalence, health effects, and support strategies. Am J Geriatr Psychiatry 2004;12:240-249.

9. Petersen RC, Doody R, Kurz A, Mohs RC, Morris JC, Rabins PV, et al. Current concepts in mild cognitive impairment. Arch Neurol 2001;58: 1985-1992.

10. Peters ME, Rosenberg PB, Steinberg M, Tschanz JT, Norton MC, Welsh-Bohmer KA, et al. Prevalence of neuropsychiatric symptoms in CIND and its subtypes: the Cache County Study. Am J Geriatr Psychiatry 2012;20:416-424.

11. Rosenberg PB, Mielke MM, Appleby B, Oh E, Leoutsakos JM, Lyketsos CG. Neuropsychiatric symptoms in MCI subtypes: the importance of executive dysfunction. Int J Geriatr Psychiatry 2011;26: 364-372.

12. Ryan KA, Weldon A, Persad C, Heidebrink JL, Barbas N, Giordani B. Neuropsychiatric symptoms and executive functioning in patients 
with mild cognitive impairment: relationship to caregiver burden. Dement Geriatr Cogn Disord 2012;34:206-215.

13. Bruce JM, McQuiggan M, Williams V, Westervelt H, Tremont G. Burden among spousal and child caregivers of patient with mild cognitive impairment. Dement Geriatr Cogn Disord 2008;25:385-390.

14. Petersen RC. Mild cognitive impairment as a diagnostic entity. J Intern Med 2004;256:183-194.

15. McKhann G, Drachman D, Folstein M, Katzman R, Price D, Stadlan EM. Clinical diagnosis of Alzheimer's disease: report of the NINCDSADRDA Work Group under the auspices of Department of Health and Human Services Task Force on Alzheimer's Disease. Neurology 1984;34:939-944.

16. Román GC, Tatemichi TK, Erkinjuntti T, Cummings JL, Masdeu JC, Garcia JH, et al. Vascular dementia: diagnostic criteria for research studies. Report of the NINDS-AIREN International Workshop. Neurology 1993;43:250-260.

17. Kang Y, Na DL. Seoul Neuropsychological Screening Battery (SNSB) Manual. Seoul: Human Brain Research \& Consulting Co., 2003.

18. Kim H, Na DL. Normative data on the Korean version of the Boston Naming Test. J Clin Exp Neuropsychol 1999;21:127-133.

19. Kang Y, Na DL, Hahn S. A validity study on the Korean Mini-Mental State Examination (K-MMSE) in dementia patients. $J$ Korean Neurol Assoc 1997; 15:300-308.

20. Hughes CP, Berg L, Danziger WL, Coben LA, Martin RL. A new clinical scale for the staging of dementia. Br J Psychiatry 1982;140: 566-572.

21. Mahoney FI, Barthel DW. Functional evaluation: the Barthel index. Md State Med J 1965;14:61-65.

22. Ku HM, Kim JH, Kwon EJ, Kim SH, Lee HS, Ko HJ, et al. A study on the reliability and validity of Seoul-Instrumental Activities of Daily Living (S-IADL). J Korean Neuropsychiatr Assoc 2004;43:189199.

23. Jung IK, Kwak DI, Shin DK, Lee MS, Lee HS, Kim JY. A reliability and validity study of Geriatric Depression Scale. J Korean Neuropsychiatr Assoc 1997;36:103-112.

24. Choi SH, Na DL, Kwon HM, Yoon SJ, Jeong JH, Ha CK. The Korean version of the neuropsychiatric inventory: a scoring tool for neuropsychiatric disturbance in dementia patients. J Korean Med Sci 2000; 15:609-615.

25. Sohn SI, Kim do H, Lee MY, Cho YW. The reliability and validity of the Korean version of the Pittsburgh Sleep Quality Index. Sleep Breath 2012;16:803-812.

26. Cho YW, Lee JH, Son HK, Lee SH, Shin C, Johns MW. The reliability and validity of the Korean version of the Epworth sleepiness scale. Sleep Breath 2011;15:377-384.

27. Bastien $\mathrm{CH}$, Vallières A, Morin CM. Validation of the Insomnia Severity Index as an outcome measure for insomnia research. Sleep Med 2001;2:297-307.

28. Netzer NC, Stoohs RA, Netzer CM, Clark K, Strohl KP. Using the
Berlin Questionnaire to identify patients at risk for the sleep apnea syndrome. Ann Intern Med 1999;131:485-491.

29. Walters AS. Toward a better definition of the restless legs syndrome. The International Restless Legs Syndrome Study Group. Mov Disord 1995; 10:634-642.

30. Cho MJ, Bae JN, Suh GH, Hahm BJ, Kim JK, Lee DW, et al. Validation of Geriatric Depression Scale, Korean Version(GDS) in the Assessment of DSM-III-R Major Depression. $J$ Korean Neuropsychiatr Assoc 1999;38:48-63.

31. Lee JY, Dong Woo Lee, Cho SJ, Na DL, Hong Jin Jeon, Kim SK, et al. Brief screening for mild cognitive impairment in elderly outpatient clinic: validation of the Korean version of the Montreal Cognitive Assessment. J Geriatr Psychiatry Neurol 2008;21:104-110.

32. Castro CM, Lee KA, Bliwise DL, Urizar GG, Woodward SH, King AC. Sleep patterns and sleep-related factors between caregiving and non-caregiving women. Behav Sleep Med 2009;7:164-179.

33. McCurry SM, Vitiello MV, Gibbons LE, Logsdon RG, Teri L. Factors associated with caregiver reports of sleep disturbances in persons with dementia. Am J Geriatr Psychiatry 2006;14:112-120.

34. Cupidi C, Realmuto S, Lo Coco G, Cinturino A, Talamanca S, Arnao $\mathrm{V}$, et al. Sleep quality in caregivers of patients with Alzheimer's disease and Parkinson's disease and its relationship to quality of life. Int Psychogeriatr 2012;24:1827-1835.

35. Creese J, Bédard M, Brazil K, Chambers L. Sleep disturbances in spousal caregivers of individuals with Alzheimer's disease. Int Psychogeriatr 2008;20:149-161.

36. McCurry SM, Pike KC, Vitiello MV, Logsdon RG, Teri L. Factors associated with concordance and variability of sleep quality in persons with Alzheimer's disease and their caregivers. Sleep 2008;31:741748.

37. von Känel R, Mausbach BT, Ancoli-Israel S, Dimsdale JE, Mills PJ, Patterson TL, et al. Sleep in spousal Alzheimer caregivers: a longitudinal study with a focus on the effects of major patient transitions on sleep. Sleep 2012;35:247-255.

38. Peng HL, Chang YP. Sleep disturbance in family caregivers of individuals with dementia: a review of the literature. Perspect Psychiatr Care 2013;49:135-146.

39. Stepanski EJ, Wyatt JK. Use of sleep hygiene in the treatment of insomnia. Sleep Med Rev 2003; 7:215-225.

40. McCurry SM, Gibbons LE, Logsdon RG, Vitiello MV, Teri L. Insomnia In Caregivers Of Persons With Dementia: Who Is At Risk And What Can Be Done About It? Sleep Med Clin 2009;4:519-526.

41. Hita-Yañez E, Atienza M, Cantero JL. Polysomnographic and subjective sleep markers of mild cognitive impairment. Sleep 2013;36: 1327-1334.

42. Brummett BH, Babyak MA, Siegler IC, Vitaliano PP, Ballard EL, Gwyther LP, et al. Associations among perceptions of social support, negative affect, and quality of sleep in caregivers and noncaregivers. Health Psychol 2006;25:220-225. 